\title{
Short-term suppression of the renin-angiotensin system in mice associated with hypertension during pregnancy
}

\author{
TOMOHIRO ISHIMARU ${ }^{1,2}$, JUNJI ISHIDA ${ }^{1,2}$, SHOKO NAKAMURA $^{1,2}$, MISUZU HASHIMOTO $^{1,2}$, \\ TANOMU MATSUKURA ${ }^{1,2}$, AYUMI NAKAMURA ${ }^{1,2}$, SATOSHI KUNITA $^{3}$, \\ FUMIHIRO SUGIYAMA $^{3}$, KEN-ICHI YAGAMI ${ }^{3}$ and AKIYOSHI FUKAMIZU ${ }^{1,2}$ \\ ${ }^{1}$ Life Science Center, Tsukuba Advanced Research Alliance; ${ }^{2}$ Graduate School of Life and Environmental Sciences, \\ University of Tsukuba, Tsukuba, Ibaraki 305-8577; ${ }^{3}$ Laboratory Animal Resource Center, \\ University of Tsukuba, Tsukuba, Ibaraki 305-8575, Japan
}

Received March 5, 2012; Accepted March 16, 2012

DOI: $10.3892 / \mathrm{mmr} .2012 .886$

\begin{abstract}
Pregnancy-induced hypertension or pre-eclampsia is a major disorder that may result in serious complications for the mother and fetus. It is characterized from maternal hypertension in late pregnancy and peripheral tissue damage, including kidney, heart and placenta, and the fetus suffers from intrauterine growth retardation (IUGR) and high perinatal mortality. Recently, it has been postulated that angiotensin II (Ang II), a potent vasoconstrictor in the renin-angiotensin system (RAS), plays a pivotal role in the pathogenesis of pre-eclampsia; however, the beneficial effect of the suppression of RAS has not yet been fully elucidated. Previously, we generated a transgenic mouse model that developed pregnancyassociated hypertension (PAH) by the overproduction of Ang II in maternal circulation during late pregnancy. In addition, mice with PAH exhibited maternal and fetal abnormalities, such as proteinuria, cardiac hypertrophy, placental morphological changes and IUGR. In this study, in order to attenuate the activity of redundant RAS during the advanced stages of PAH, we administered olmesartan $(\mathrm{Olm})$, an angiotensin receptor blocker, and captopril (Cp), an angiotensin converting enzyme inhibitor, from E17 to E19 days of gestation, and evaluated its effect on cardiac and placental abnormalities and fetal growth. $\mathrm{Olm}$ and $\mathrm{Cp}$ administration significantly lowered the blood pressure of mice with $\mathrm{PAH}$, and placental histological change and severe IUGR were markedly ameliorated in both groups. On the contrary, Olm or Cp treatment had little effect on cardiac remodeling during the advanced stages of PAH. These findings highlight a variety of therapeutic actions of RAS repression on the progressive pathology of PAH in mice.
\end{abstract}

Correspondence to: Dr Akiyoshi Fukamizu, Life Science Center, Tsukuba Advanced Research Alliance, University of Tsukuba, Tsukuba, Ibaraki 305-8577, Japan

E-mail: akif@tara.tsukuba.ac.jp

Key words: pregnancy, hypertension, pre-eclampsia, renin-angiotensin system, transgenic mice

\section{Introduction}

Each year, 536,000 women die from pregnancy complications and childbirth (1). Hypertensive disorders are the most common complications in pregnancy. Pregnancy-induced hypertension or pre-eclampsia is defined as the presence of hypertension and proteinuria in the second or third trimester of pregnancy (2), and leads to complications in approximately $10 \%$ of pregnancies in humans. Mortality rates in mothers with pre-eclampsia are as high as $30 \%$, and perinatal mortality is between 6.6 and 60\% (3-5). The prevention or improvement of pre-eclampsia would have a significant impact on maternal and neonatal outcome, but the molecular basis of the pathogenesis remains unclear.

The renin (RN)-angiotensin system (RAS) is a major determinant of blood pressure and sodium balance under usual conditions as well as in pregnancy $(6,7)$. The reaction between $\mathrm{RN}$ as an aspartyl protease and its substrate, angiotensinogen (ANG), generates angiotensin I. It is further processed into the potent vasopressor angiotensin II (Ang II) by the angiotensinconverting enzyme (ACE) (8). Two Ang II receptors have been identified in humans: AT1 (type I) and AT2 (type II). Rodents, on the contrary, express type 1a (AT1a) and type 1b (AT1b), in which blood pressure and the pathophysiological effects of RAS are primarily mediated by the ATla receptor (9).

In normal pregnancy, plasma levels of Ang II are elevated in association with increased ANG and plasma RN activity during gestation $(10,11)$. Normal pregnant women are resistant to the pressor effects of Ang II (12-14), and they remain normotensive. On another front, vascular sensitivity to Ang II is elevated in pre-eclamptic women (13). It has been reported that there are several pathways that potentially enhance AT1-mediated signaling in pre-eclampsia $(15,16)$. However, the direct correlation between RAS activation and the pathophysiological mechanisms of pre-eclampsia remain unknown.

We previously generated mice with pregnancy-associated hypertension (PAH) by mating females expressing human angiotensinogen (hANG) with males expressing human renin (hRN) (17). In mice with PAH, maternal hypertension begins at 14 days of gestation (E14) until delivery (E20), due to the generation of excessive Ang I by hRN secretion from the fetus 
into the maternal circulation $(17,18)$. Mice with PAH have also been shown to exhibit proteinuria and cardiac hypertrophy, and fetuses at term have shown severe intrauterine growth retardation (IUGR) with placental morphological changes (18-20). We have previously demonstrated the importance of the AT1 receptor in the pathogenesis of hypertension during pregnancy using mice with PAH $(18,21)$; however, the beneficial effect of RAS suppression during the advanced stages of PAH was not fully elucidated. In the present study, we administered olmesartan (Olm), an angiotensin receptor blocker (ARB), and captopril (Cp), an ACE inhibitor, from the 17th day of gestation (E17) to the 19th day of gestation (E19), in order to attenuate the activity of redundant RAS during the progressive stage of $\mathrm{PAH}$, and evaluated its effect on physiological changes during pregnancy and tissue damages at E19.

\section{Materials and methods}

Animals. As described previously, mice with PAH were generated by mating females expressing hANG with males expressing hRN (17). Transgenic mice and age-matched wild-type mice (C57BL/6J) at 10-20 weeks of age were used for cross-mating. Animal experiments were carried out in a humane manner under approval from the Institutional Animal Experiment Committee of the University of Tsukuba (Tsukuba, Japan). Experiments were performed in accordance with the Regulation of Animal Experiments of the University of Tsukuba and the Fundamental Guidelines for Proper Conduct of Animal Experiments and Related Activities in Academic Research Institutions under the jurisdiction of the Ministry of Education, Culture, Sports, Science and Technology.

Administration of ARB and ACE inhibitors. Olm was a gift from Daiichi Sankyo Co., Ltd. (Tokyo, Japan). Olm was dissolved in sterile water containing $0.1 \%$ (weight/volume) $\mathrm{NaHCO}_{3}$ and $0.1 \%$ (weight/volume) $\mathrm{KHCO}_{3}$ and administered at a dose of $15 \mathrm{mg} / \mathrm{l}$ in drinking water from E17 to the 18th day of gestation (E18), and at a dose of $50 \mathrm{mg} / 1$ from E18 to E19 in drinking water. Cp was administered at a dose of $250 \mathrm{mg} / \mathrm{l}$ in drinking water. These dosages were determined by preliminary experiments to bring the systolic blood pressure (SBP) of mice with PAH to $90-120 \mathrm{mmHg}$. Female mice were classified into four groups: i) wild-type mice, ii) mice with $\mathrm{PAH}$, iii) Olm-treated mice with $\mathrm{PAH}$ and iv) $\mathrm{Cp}$-treated mice with $\mathrm{PAH}$.

Measurement of blood pressure and body weight. SBP was measured in awake animals [each group, $n=4-9$; except on the 9th day of gestation (E9) in Olm-treated mice with PAH $(n=3)]$ using a non-invasive computerized tail-cuff blood pressure system (BP-98a; Softron, Tokyo, Japan) as described previously (18). During measurement, unanesthetized mice were guided into a holder mounted on a thermostatically controlled warming plate and maintained at $37^{\circ} \mathrm{C}$. Body weight was measured on the 1st day of gestation (E1) and from E9 to E19 (each group, $\mathrm{n}=5-8$ ).

Histological analysis. Female mice were euthanized by bleeding under deep anesthesia on E19. The extracted fetuses were photographed and their body weight was measured. The maternal hearts and placentae were immediately excised, fixed with $10 \%$ neutral-buffered formalin solution and observed under a stereomicroscope (SZX2-ILLT; Olympus, Japan). After being sufficiently washed in PBS, tissues were dehydrated and embedded in paraffin wax. Placental sections were cut to $5-\mu \mathrm{m}$ thickness and heart sections were cut to $3 \mu \mathrm{m}$ using a rotary microtome (Microm HM340E; Walldorf, Germany). To evaluate cardiac fibrosis, heart sections were stained with Masson's trichrome. For morphological observation, placental sections were stained with H\&E. Stained sections were examined under a light microscope (BX53; Olympus).

Statistical analysis. Statistical comparison was performed using GraphPad Prism version 5 for Macintosh (GraphPad Software, San Diego, CA, USA). The Student's t-test, Welch's t-test, or the Mann-Whitney U test were adapted for the evaluation of the significance of the differences between groups. $\mathrm{P}<0.05$ denoted a statistically significant difference. Results are expressed as the means $\pm \mathrm{SE}$.

\section{Results}

Effect of short-term administration of Olm or Cp on physiological alterations in mice with PAH. First, we examined the effectiveness of short-term $\mathrm{Olm}$ or $\mathrm{Cp}$ administration on PAH pathology in pregnant mice. The SBP remained unchanged during pregnancy in normal pregnant (wild-type) mice (Fig. 1A, open circle), but began to increase in mice with PAH from 14 days of gestation (E14), reaching a peak on E18 (Fig. 1A, closed circle). Therefore, we administered Olm or Cp to mice with PAH from E17 to E19 at the progressive stage of hypertension. Olm and $\mathrm{Cp}$ significantly lowered the blood pressure of mice with PAH to the normal range (Fig. 1A, open and closed square, respectively). Mice with $\mathrm{PAH}$ displayed a significant suppression of weight gain during late pregnancy (Fig. 1B, closed circle) in comparison to wild-type mice (Fig. 1B, opened circle). The weight gain of Olm- and $\mathrm{Cp}$-treated mice with PAH was partially recovered (Fig. 1B, open and closed square, respectively). These findings indicate that the suppression of RAS activity by $\mathrm{Cp}$ treatment effectively reduces blood pressure and restores weight gain in mice with PAH. Olm treatment also improves the abnormalities in blood pressure and weight gain in mice with $\mathrm{PAH}$.

Ineffectiveness of Olm or Cp treatment on cardiac remodeling in mice with PAH. Gross observation of the E19 hearts of mice with $\mathrm{PAH}$ revealed prominent hypertrophy compared to wild-type mice (Fig. 2A upper panels). In addition, the heart weight of E19 mice with PAH was significantly increased compared to wild-type pregnant mice (Fig. 2B, black bar). These hypertrophic changes in mice with $\mathrm{PAH}$ were not regressed by Olm or $\mathrm{Cp}$ administration (Fig. 2A, lower panel and Fig. 2B, striped or tinted bar). Increased heart weight with hypertension is often accompanied by cardiac remodeling, including ventricular hypertrophy and fibrosis, which further aggravate cardiac dysfunction. Therefore, we investigated in detail the pathological changes and effects of RAS suppression on the hearts of mice with PAH. Heart sections from mice with PAH on E19 exhibited ventricular hypertrophy compared to sections from wild-type mice (Fig. 2C, upper panel). This ventricular morphological change in mice with $\mathrm{PAH}$ was little 

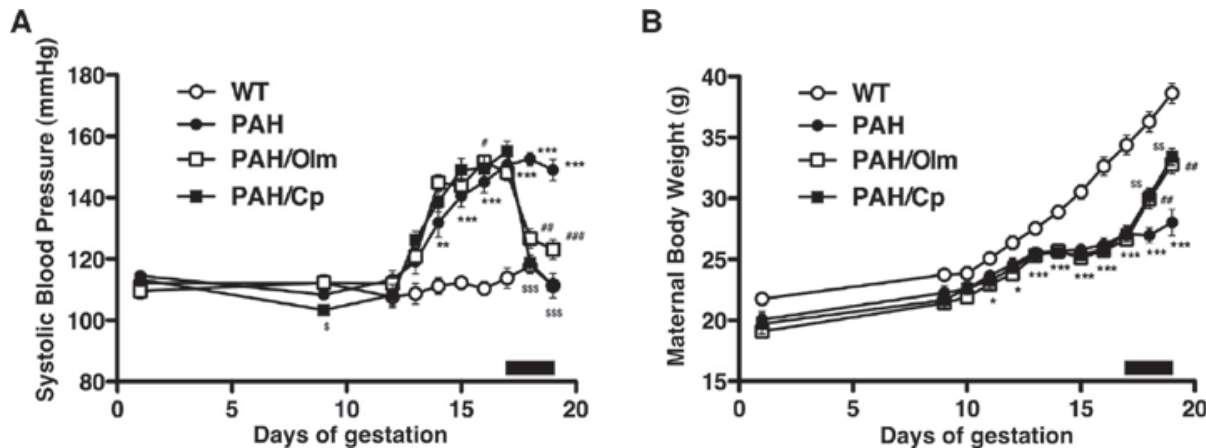

Figure 1. Amelioration of hypertension and normalization of weight gain in mice with PAH by Olm or Cp treatment from E17 to E19. (A) Systolic blood pressure during pregnancy in wild-type (WT) mice, non-treated mice with PAH, Olm-treated mice with PAH and Cp-treated mice with PAH. The heavy horizontal line at the end of the chart indicates the duration of drug administration. In mice with $\mathrm{PAH},{ }^{* *} \mathrm{P}<0.01$ and ${ }^{* * * *} \mathrm{P}<0.001$ vs. WT mice. In Olm-treated mice with $\mathrm{PAH},{ }^{\#} \mathrm{P}<0.05,{ }^{\# \#} \mathrm{P}<0.01$ and ${ }^{\# \# \#} \mathrm{P}<0.001$ vs. mice with $\mathrm{PAH}$. In $\mathrm{Cp}$-treated mice with $\mathrm{PAH},{ }^{\mathrm{S}} \mathrm{P}<0.05$ and ${ }^{\$ \$ S} \mathrm{P}<0.001$ vs. mice with $\mathrm{PAH}$. (B) Changes in maternal body weight during pregnancy of WT mice, non-treated mice with PAH, Olm-treated mice with PAH and Cp-treated mice with PAH. Each group, $\mathrm{n}=5-8$. The heavy horizontal line at the end of the chart indicates the duration of drug administration. In mice with $\mathrm{PAH},{ }^{*} \mathrm{P}<0.05$ and ${ }^{* * *} \mathrm{P}<0.001$ vs. WT mice. In Olm-treated mice with $\mathrm{PAH},{ }^{\# \#} \mathrm{P}<0.01$ vs. mice with $\mathrm{PAH}$. In $\mathrm{Cp}$-treated mice with $\mathrm{PAH},{ }^{\$ \$} \mathrm{P}<0.01$ vs. mice with PAH.

improved by Olm or Cp (Fig. 2C, upper panel). Additionally, cardiac fibrosis was not observed in wild-type mice, but mice with PAH showed remarkable myocardial interstitial fibrosis (Fig. 2C, lower panel) that was unchanged by Olm or Cp administration. These results indicate that the short-term suppression of RAS activity during late pregnancy is ineffective on the Ang II-induced cardiac remodeling in mice with PAH.

Improvement of placental abnormalities in mice with PAH by Olm or Cp treatment. Pre-eclampsia is also associated with placental and fetal defects in humans. We then examined the feto-placental abnormalities in mice with PAH. In the mice with PAH, the gross morphology of the fetal side of the placenta on E19 showed a decrease in vascularization compared to that in wild-type mice, and this was, at least in part, restored by Olm or Cp administration (Fig. 3A). Moreover, morphological changes, including edematous enlargement, were found in $\mathrm{PAH}$ labyrinth at E19 as reported in our previous studies (18-20). This histological change observed in the placentae from mice with PAH was partially abated by Olm or Cp treatment (Fig. 3B).

Recovery of IUGR in PAH fetus by Olm or Cp treatment. IUGR of the fetus occurs in many patients with pre-eclampsia, which is often associated with placental morphological changes. Therefore, we investigated the fetal defects in mice with PAH. The fetuses from mice with PAH showed severe IUGR, and the mean fetal body weight was $~ 73 \%$ of that from wild-type mice (Fig. 4A, upper panel and Fig. 4B, black bar). Furthermore, the weights of the fetuses on E19 had significantly recovered and matched the weights of the wild-type mice by the short-term administration of Olm and Cp (mean fetal body weight $91 \%$ and $91 \%$ of that from wild-type mice, respectively) (Fig. 4B, striped or tinted bar). These results indicate that the suppression of the enhanced RAS activation in the last 2 days of pregnancy has the ability to dramatically improve the fetal growth retardation in mice with $\mathrm{PAH}$.

\section{Discussion}

RAS plays an important role in the maintenance of homeostasis in normal pregnancy. Blood Ang II levels increase as pregnancy progresses (22) to achieve the adequate amount of fluid volume for feto-placental circulation. The systemic RAS of pre-eclamptic mothers is suppressed, but not activated (23), even so, the vascular sensitivity for Ang II is elevated in preeclamptic patients (13). In addition, AbdAlla et al (15) showed that the bradykinin B2 receptor protein level increased in preeclamptic patients and that AT1-bradykinin B2 heterodimers accelerated AT1-mediated signal transduction. On the contrary, Wallukat et al (16) reported that agonistic autoimmune antibody against AT1 was detected in the sera of pre-eclamptic women. These data show evidence of the deteriorative action of Ang II on the pathogenesis of pre-eclampsia; however, it has been difficult to elucidate the pathophysiological significance of aberrant RAS activation in the human case, because of the contraindication of RAS inhibition during pregnancy $(24,25)$.

Previously, using mice with $\mathrm{PAH}$, we demonstrated the importance of RAS activation with enhanced AT1 signaling in the pathogenesis of hypertension during pregnancy $(18,21)$. Additionally, the short-term administration of an ARB for the last 2 days of pregnancy in mice with PAH also recovered IUGR (18); however, the therapeutic but not preventive effect of RAS suppression on extensive PAH pathology was not clarified. In this study, we administered Olm and $\mathrm{Cp}$ to mice with $\mathrm{PAH}$ from E17 to E19. SBP was decreased to the levels of wild-type mice and the suppression of weight gain during late pregnancy was recovered in both treated PAH groups (Fig. 1A and B), suggesting that excess RAS activation in mice with PAH was successfully suppressed. Therefore, we investigated the ameliorative effect of the short-term repression of RAS activity on the cardiac and feto-placental abnormalities in mice with PAH.

Cardiac hypertrophy is the major outcome of cardiac remodeling, and AT1-mediated signaling, including the vasopressor action, has been implicated $(26,27)$. In addition, cardiac fibrosis is a critical risk factor for cardiac dysfunction accompanied by cardiac remodeling, and a number of studies have shown that RAS activation is thought to contribute to the development of cardiac fibrosis (28-31). Notably, in the present study, cardiac hypertrophy and severe fibroses in mice with $\mathrm{PAH}$ were not improved by the short-term administration of Olm or $\mathrm{Cp}$ (Fig. 2A-C). These results are not consistent with our previous finding that, in mice with $\mathrm{PAH}$, an ARB 
A

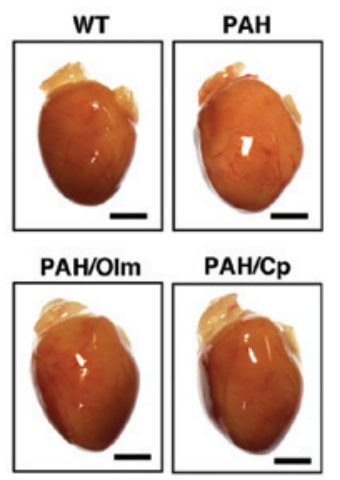

B

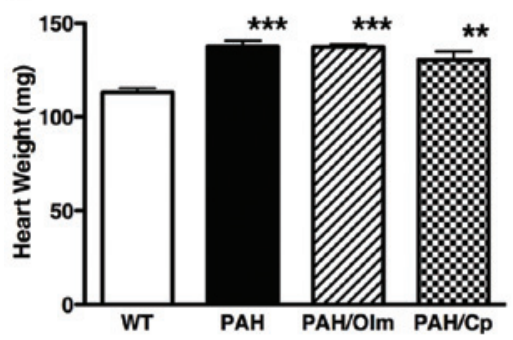

C

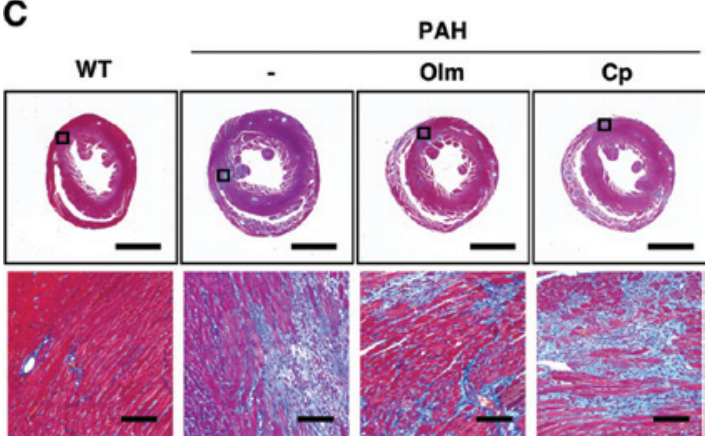

Figure 2. Evaluation of cardiac hypertrophy and remodeling. (A) Gross observation of the heart on E19. Scale bar, $2 \mathrm{~mm}$. (B) Heart weight in pregnant mice at E19 ( $n=6-13)$. Each heart weight is represented by wet weight. ${ }^{* *} \mathrm{P}<0.01$ and ${ }^{* * *} \mathrm{P}<0.001$ vs. WT mice. (C) Upper panels show Masson's trichrome staining of the cross-sectional aspects of the heart. Scale bar, $2 \mathrm{~mm}$. Lower panels show higher magnifications of corresponding squares shown in the upper panels. Scale bar, $100 \mu \mathrm{m}$.

treatment from the 13th day of gestation (E13) to E19 attenuated the cardiac abnormalities, including hypertrophy and remodeling. This indicates that the aberrant RAS activation in the advanced stages of PAH plays a critical role in the development of maternal hypertension, but is inefficient in the worsened cardiac remodeling in mice with PAH.

It has been suggested that pre-eclampsia is also associated with placental and fetal defects in humans, and there is involvement of RAS activation in the development of feto-placental abnormalities. In pre-eclamptic patients, AT1 is up-regulated in the placenta (32), and the expression of AT1 increases in the placenta of endotoxin-induced hypertension in pregnant rats (33). The abnormal balance of vasoactive factors was also revealed in pre-eclamptic pregnant patients. The solublefms-like tyrosine kinase-1 (sFlt-1) is an endogenous inhibitor of vascular endothelial growth factor (VEGF) and placental growth factor (PlGF), and high levels of circulating sFlt-1 cause
A

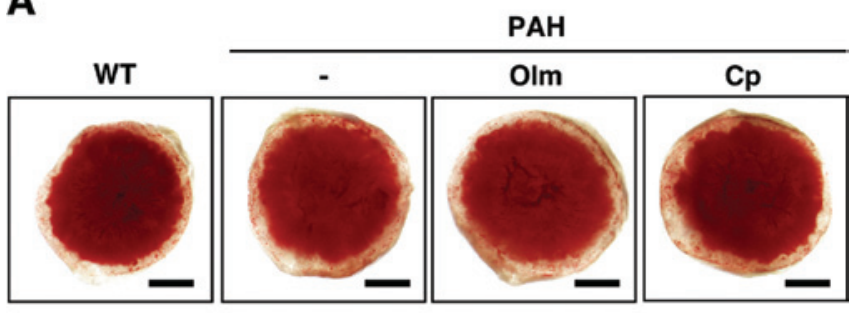

B

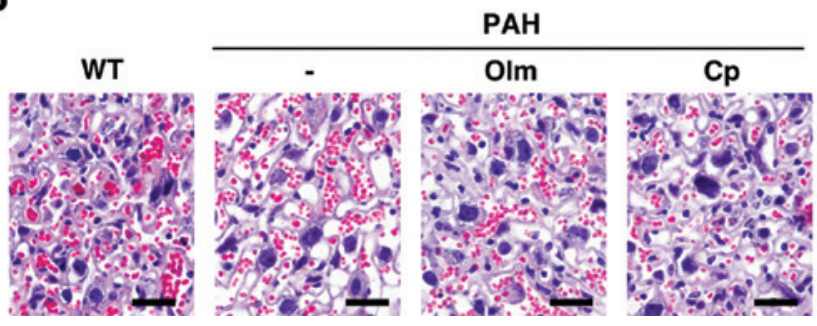

Figure 3. Histopathological analysis of the placentae at E19. (A) Gross observation of the placentae on E19. Scale bar, $2 \mathrm{~mm}$. (B) H\&E staining of the cross-sectional aspects of the labyrinth. Scale bar, $30 \mu \mathrm{m}$.
A

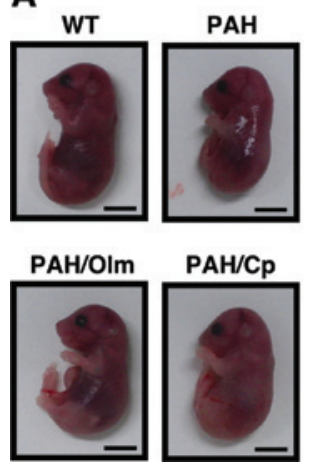

B

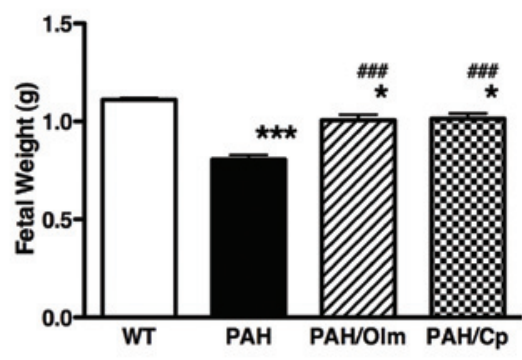

Figure 4. Improvement of IUGR in mice with PAH by Olm or Cp treatment. (A) Gross observation of fetuses on E19. Scale bar, $5 \mathrm{~mm}$. (B) Fetal body weight at E19 ( $\mathrm{n}=6-14$ of dams). Each fetal body weight is represented by wet weight. ${ }^{*} \mathrm{P}<0.05$ and ${ }^{* * *} \mathrm{P}<0.001$ vs. WT mice, and ${ }^{\# \# \#} \mathrm{P}<0.001$ vs. mice with $\mathrm{PAH}$.

widespread dysfunction of endothelial cells by interfering with the action of VEGF and PlGF $(34,35)$. Kumasawa et al $(36)$ demonstrated that lentiviral vector-mediated placenta-specific expression of sFlt-1 caused maternal hypertension, proteinuria and IUGR in mice. It has also been reported that Ang II significantly elevated circulating levels of sFlt-1 in pregnant mice (37). The plasma levels of sFlt-1 in mice with PAH were significantly increased at E19 (20). In this study, at E19 of mice with $\mathrm{PAH}$, morphological changes, including edematous enlargement, were found in the labyrinth (Fig. 3A and B) and severe IUGR was observed in the fetuses (Fig. 4A and B), as shown in our previous study $(18,19)$. These abnormalities were improved in part by short-term treatment of Olm as well as Cp (Figs. 3 and 4). This indicates that the aberrant RAS activation in the last term of pregnancy greatly contributes to the aggravation of feto-placental abnormalities, and suggests that the restored placental structure by $\mathrm{Olm}$ or $\mathrm{Cp}$ treatment changes the fetal IUGR to a favorable up-growth.

In conclusion, using mice with PAH treated with shortterm administration of $\mathrm{Olm}$ or $\mathrm{Cp}$, we demonstrated that 
the suppression of aberrant RAS activation only for 2 days before delivery during the advanced stages of PAH effectively improved severe maternal hypertension and feto-placental defects, including IUGR, but was ineffective in exacerbated cardiac remodeling. The findings from our study using mice provide insight into the effectiveness of inhibiting RAS activity, thus alleviating some of the extensive symptoms in pregnancy-associated hypertension.

\section{Acknowledgements}

The authors thank Daiichi Sankyo Co., Ltd., Tokyo, Japan, for the gift of olmesartan. They are grateful to the members of the Fukamizu Laboratory for their helpful discussions and encouragement. This study was supported by the following grants: A Grant-in-Aid for Scientific Research (B), a Grantin-Aid for Young Scientists (B), and a Grant-in-Aid for JSPS Fellows from the Ministry of Education, Culture, Sports, Science and Technology of Japan.

\section{References}

1. WHO U, UNFPA and World Bank: Maternal Mortality in 2005: Estimates Developed by WHO, UNICEF, UNFPA and the World Bank. WHO, Geneva, 2007.

2. Report of the National High Blood Pressure Education Program Working Group on High Blood Pressure in Pregnancy. Am J Obstet Gynecol 183: S1-S22, 2000.

3. Weinstein L: Syndrome of hemolysis, elevated liver enzymes, and low platelet count: a severe consequence of hypertension in pregnancy. Am J Obstet Gynecol 142: 159-167, 1982.

4. Sibai BM, Ramadan MK, Usta I, Salama M, Mercer BM and Friedman SA: Maternal morbidity and mortality in 442 pregnancies with hemolysis, elevated liver enzymes, and low platelets (HELLP syndrome). Am J Obstet Gynecol 169: 1000-1006, 1993.

5. Vigil-De Gracia P: Maternal deaths due to eclampsia and HELLP syndrome. Int J Gynaecol Obstet 104: 90-94, 2009.

6. August P, Lenz T, Ales KL, et al: Longitudinal study of the renin-angiotensin-aldosterone system in hypertensive pregnant women: deviations related to the development of superimposed preeclampsia. Am J Obstet Gynecol 163: 1612-1621, 1990.

7. Brown MA, Nicholson E and Gallery ED: Sodium-reninaldosterone relations in normal and hypertensive pregnancy. $\mathrm{Br} \mathrm{J}$ Obstet Gynaecol 95: 1237-1246, 1988.

8. Oparil S and Haber E: The renin-angiotensin system (first of two parts). N Engl J Med 291: 389-401, 1974.

9. Crowley SD, Tharaux PL, Audoly LP and Coffman TM: Exploring type I angiotensin (AT1) receptor functions through gene targeting. Acta Physiol Scand 181: 561-570, 2004.

10. Brown MA, Wang J and Whitworth JA: The renin-angiotensinaldosterone system in pre-eclampsia. Clin Exp Hypertens 19 713-726, 1997.

11. Baker PN, Broughton Pipkin F and Symonds EM: Platelet angiotensin II binding and plasma renin concentration, plasma renin substrate and plasma angiotensin II in human pregnancy. Clin Sci (Lond) 79: 403-408, 1990.

12. Chesley LC, Talledo E, Bohler CS and Zuspan FP: Vascular reactivity to angiotensin II and norepinephrine in pregnant women. Am J Obstet Gynecol 91: 837-842, 1965.

13. Gant NF, Daley GL, Chand S, Whalley PJ and MacDonald PC: A study of angiotensin II pressor response throughout primigravid pregnancy. J Clin Invest 52: 2682-2689, 1973.

14. Baker PN, Broughton Pipkin F and Symonds EM: Comparative study of platelet angiotensin II binding and the angiotensin II sensitivity test as predictors of pregnancy-induced hypertension. Clin Sci (Lond) 83: 89-95, 1992

15. AbdAlla S, Lother H, el Massiery A and Quitterer U: Increased AT(1) receptor heterodimers in preeclampsia mediate enhanced angiotensin II responsiveness. Nat Med 7: 1003-1009, 2001.
16. Wallukat G, Homuth V,Fischer T, et al: Patients with preeclampsia develop agonistic autoantibodies against the angiotensin AT1 receptor. J Clin Invest 103: 945-952, 1999.

17. Takimoto E, Ishida J, Sugiyama F, Horiguchi H, Murakami K and Fukamizu A: Hypertension induced in pregnant mice by placental renin and maternal angiotensinogen. Science 274: 995-998, 1996.

18. Saito T, Ishida J, Takimoto-Ohnishi E, et al: An essential role for angiotensin II type 1a receptor in pregnancy-associated hypertension with intrauterine growth retardation. FASEB J 18: 388-390, 2004.

19. Takimoto-Ohnishi E, Saito T, Ishida J, et al: Differential roles of renin and angiotensinogen in the feto-maternal interface in the development of complications of pregnancy. Mol Endocrinol 19: 1361-1372, 2005

20. Furuya M, Ishida J, Inaba S, et al: Impaired placental neovascularization in mice with pregnancy-associated hypertension. Lab Invest 88: 416-429, 2008.

21. Sakairi A, Ishida J, Honjo K, et al: Angiotensin type 1 receptor blockade prevents cardiac remodeling in mice with pregnancyassociated hypertension. Hypertens Res 31: 2165-2175, 2008.

22. Zheng J, Bird IM, Chen DB and Magness RR: Angiotensin II regulation of ovine fetoplacental artery endothelial functions: interactions with nitric oxide. J Physiol 565: 59-69, 2005.

23. Hanssens M, Keirse MJ, Spitz B and van Assche FA: Angiotensin II levels in hypertensive and normotensive pregnancies. Br J Obstet Gynaecol 98: 155-161, 1991

24. Shotan A, Widerhorn J, Hurst A and Elkayam U: Risks of angiotensin-converting enzyme inhibition during pregnancy: experimental and clinical evidence, potential mechanisms, and recommendations for use. Am J Med 96: 451-456, 1994.

25. Saji H, Yamanaka M, Hagiwara A and Ijiri R: Losartan and fetal toxic effects. Lancet 357: 363, 2001.

26. Hein L, Stevens ME, Barsh GS, Pratt RE, Kobilka BK and Dzau VJ: Overexpression of angiotensin AT1 receptor transgene in the mouse myocardium produces a lethal phenotype associated with myocyte hyperplasia and heart block. Proc Natl Acad Sci USA 94: 6391-6396, 1997.

27. Paradis P, Dali-Youcef N, Paradis FW, Thibault G and Nemer M: Overexpression of angiotensin II type I receptor in cardiomyocytes induces cardiac hypertrophy and remodeling. Proc Natl Acad Sci USA 97: 931-936, 2000.

28. Crabos M, Roth M, Hahn AW and Erne P: Characterization of angiotensin II receptors in cultured adult rat cardiac fibroblasts. Coupling to signaling systems and gene expression. J Clin Invest 93: 2372-2378, 1994.

29. Harada K, Sugaya T, Murakami K, Yazaki Y and Komuro I: Angiotensin II type 1A receptor knockout mice display less left ventricular remodeling and improved survival after myocardial infarction. Circulation 100: 2093-2099, 1999.

30. Lijnen PJ, Petrov VV and Fagard RH: Angiotensin II-induced stimulation of collagen secretion and production in cardiac fibroblasts is mediated via angiotensin II subtype 1 receptors. J Renin Angiotensin Aldosterone Syst 2: 117-122, 2001.

31. Tokuda K, Kai H, Kuwahara F, et al: Pressure-independent effects of angiotensin II on hypertensive myocardial fibrosis. Hypertension 43: 499-503, 2004.

32. Leung PS, Tsai SJ, Wallukat G, Leung TN and Lau TK: The upregulation of angiotensin II receptor AT(1) in human preeclamptic placenta. Mol Cell Endocrinol 184: 95-102, 2001.

33. Doering TP, Haller NA, Montgomery MA, Freeman EJ and Hopkins MP: The role of AT1 angiotensin receptor activation in the pathogenesis of preeclampsia. Am J Obstet Gynecol 178: 1307-1312, 1998

34. Kendall RL and Thomas KA: Inhibition of vascular endothelial cell growth factor activity by an endogenously encoded soluble receptor. Proc Natl Acad Sci USA 90: 10705-10709, 1993.

35. Stepan H, Faber R, Dornhofer N, Huppertz B, Robitzki A and Walther T: New insights into the biology of preeclampsia. Biol Reprod 74: 772-776, 2006.

36. Kumasawa K, Ikawa M, Kidoya $\mathrm{H}$, et al: Pravastatin induces placental growth factor (PGF) and ameliorates preeclampsia in a mouse model. Proc Natl Acad Sci USA 108: 1451-1455, 2011.

37. Zhou CC, Ahmad S, Mi T, et al: Angiotensin II induces soluble fms-Like tyrosine kinase-1 release via calcineurin signaling pathway in pregnancy. Circ Res 100: 88-95, 2007. 